\title{
Advantages of Direct Detection and Electron Counting for Electron Energy Loss Spectroscopy Data Acquisition and the Quest of Extremely High-Energy Edges Using Eels
}

\author{
Paolo Longo ${ }^{1}$, Jamie L. Hart ${ }^{2}$, Andrew C. Lang ${ }^{2}$, Ray D. Twesten ${ }^{1}$ and Mitra L. Taheri ${ }^{2}$ \\ ${ }^{1 .}$ Gatan Inc., 5794 W Las Positas Blvd, Pleasanton CA, USA \\ ${ }^{2}$.Department of Materials Science and Engineering, Drexel University, Philadelphia, PA, USA
}

Transmission electron microscopes primarily employ indirect cameras (IDC) for electron detection in imaging, diffraction and EELS modes. Such cameras convert incident electrons to photons which, through a fiber optic network or lens, are coupled to a light sensitive camera. This indirect detection method typically has a negative impact on the point spread function (PSF) and detective quantum efficiency (DQE) of the camera. Over the last decade, radiation tolerant CMOS active pixel sensors, which directly detect high-energy incident electrons and have the speed to count individual electrons events, have been developed. These detectors result in greatly improved PSF and DQE in comparison to conventional IDCs. Such direct detection cameras (DDCs) have revolutionized the cryo-TEM field as well as have strong advantages for in-situ TEM in both imaging and diffraction applications. EELS applications can benefit from the improved PSF and the ability to count electrons. The improved PSF allows spectra to be acquired over larger energy ranges while maintaining sharp features and greatly reduced spectral tails. The ability to count electrons nearly eliminates the noise associated with detector readout and greatly reduces the proportional noise associated with detector gain variations. This effectively leaves the shot noise as the limiting noise source present. The implication for EELS acquisition is that fine structure analysis becomes more straightforward for typical conditions and even possible for the case of low signal levels.

As example of the advantages due the reduced noise and PSF, Figures 1 show the EELS spectra extracted from the $\mathrm{Ti} \mathrm{L}_{2,3}$-edges at $456 \mathrm{eV}$ and $\mathrm{Sr} \mathrm{L}_{2,3}$-edges at $1940 \mathrm{eV}$. Here both the DD and IDC detectors were set in such way that the resulting energy range is about $2000 \mathrm{eV}$ and this can be achieved specifically with a dispersion of $1 \mathrm{eV} /$ channel in the case of IDC and $0.5 \mathrm{eV} /$ channel for DD. The DD detector is made of $4 \mathrm{k} \times 4 \mathrm{k}$ pixels, hence the use of dispersion $0.5 \mathrm{eV} /$ channel to generate a spectrum with an energy range of $2000 \mathrm{eV}$. With such high energy range both the Ti L- and Sr L-edges can be collected in the same spectrum. In the case of DD, given the much reduced PSF, the energy resolution is such that the eg and $\mathrm{t} 2 \mathrm{~g}$ peaks in both L2 and L3 edges can be easily resolved and show that the Ti is in $4+$ oxidation state. In the case of IDC, dispersion $1 \mathrm{eV} /$ channel does not allow enough energy resolution to resolve all the features in the Ti L and identify the oxidation state. Higher dispersion would be needed in order to cope with the high PSF and give enough energy resolution for chemical analysis. The much lower noise of the DD allows the collection of a spectrum where both the L2 and L3 edges of the Sr L can be easily resolved. In the case of the spectrum extracted using the IDC detector, the amount of noise is such that L2 and L3 edges cannot be resolved as nicely as in the DD case. All these data was acquired using exactly the same experimental conditions across similar areas in the same specimen.

Very high-energy edges have always been very hard or almost impossible to acquire using EELS due to the very limited amount of signal. With the introduction of DD detectors the amount of noise has been enormously reduced and as result low intensity signals can now be observed and detected. Figures 2 show EELS spectra of $\mathrm{Cu} \mathrm{K}$ and $\mathrm{N} \mathrm{K}$-edges at about $9 \mathrm{keV}$ and $8.3 \mathrm{keV}$. The spectra can be easily observed and the quality is such that high contrast elemental maps can be generated as shown in Figure 
2c. Until now, such high energy edges have been collected using synchrotron based techniques such as XAS with very limited spatial resolution. Now, by acquiring EELS data in counting mode using DD detectors, high energy edges can be collected and their signal mapped out with high spatial resolution. A new world is about to open up.

In this presentation, we will review the current state of electrons counting detectors for electron microscopy with an emphasis on system for EELS measurements.
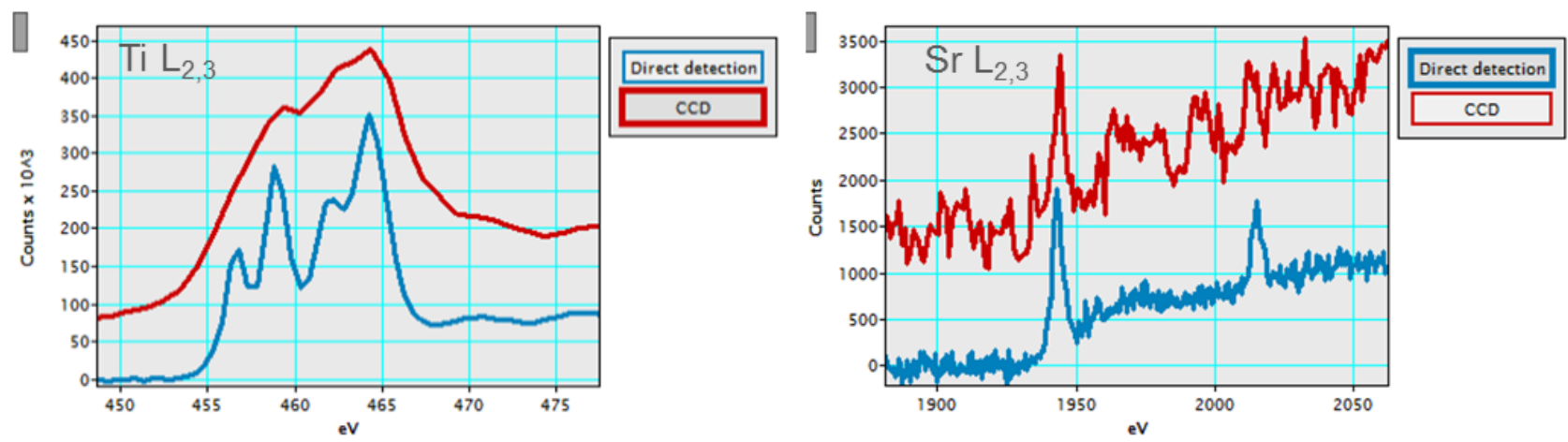

Figure 1. a,b) EELS spectra of Ti L- and Sr-edges at $456 \mathrm{eV}$ and $1940 \mathrm{eV}$ extracted from the same area in the specimen under the same experimental conditions using both the DD and IDC detectors. Both the Ti and Sr L signals are extracted from the same spectrum. The spectra extracted using the DD detector show higher energy resolution as well as much reduced noise that allows the clear observation all the main features in the near edge fine structure
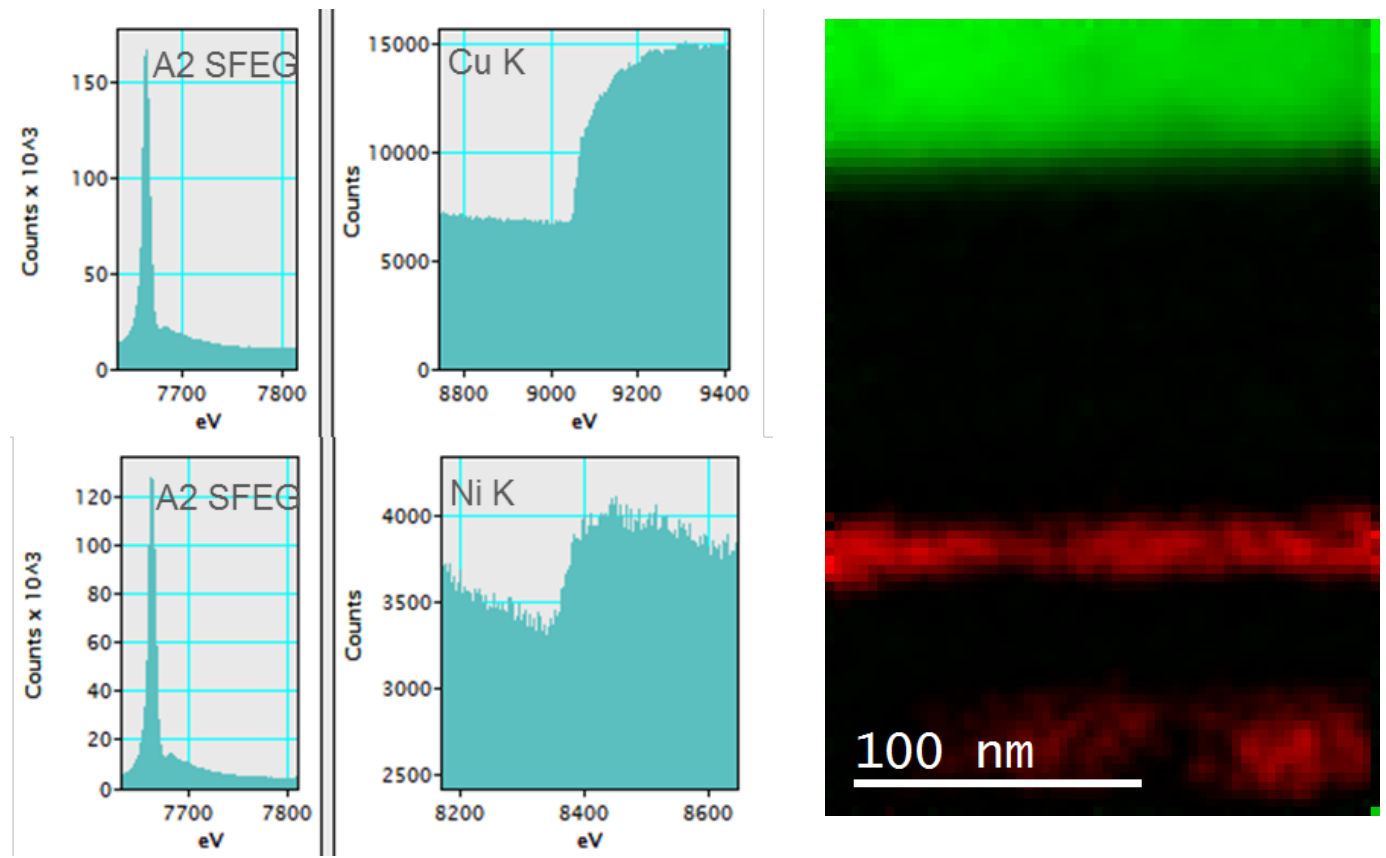

Figure 2. a,b) EELS spectra of $\mathrm{Cu} \mathrm{K}$ and $\mathrm{Ni} \mathrm{K}$ at $9 \mathrm{keV}$ and $8.3 \mathrm{keV}$ acquired in STEM mode using the DD detector in counting mode. The signal-to-noise ratio is quite and allows the clear observation. C) EELS elemental maps of $\mathrm{Ni} \mathrm{K}$ in red and $\mathrm{Cu} \mathrm{K}$ in green. This proves that such high energy edges can be collected, observed and the signals extracted generating high-contrast elemental maps in STEM mode. 